\title{
PATRONES DE VARIACIÓN DE LA DENSIDAD DE LA MADERA Y MORFOMETRÍA CELULAR DE Salix babylonica PARA LA DETERMINACIÓN DE LA EDAD DE TRANSICIÓN ENTRE MADERA JUVENIL Y MADURA
}

\section{PATTERNS OF VARIATION OF WOOD DENSITY AND CELLULAR MORPHOMETRY IN Salix babylonica FOR DETERMINING THE AGE OF TRANSITION BETWEEN JUVENILE AND MATURE WOOD}

\author{
Ana C. Cobas ${ }^{1,3,4}$, María C. Area ${ }^{2,3}$, Silvia Monteoliva ${ }^{1,3}$
}

\section{RESUMEN}

El objetivo del trabajo fue analizar los patrones de variación axial y radial de la densidad y morfometría celular de la madera de sauce americano y determinar la edad de transición de madera juvenil a madura. Se muestrearon cinco árboles de 45 años de Salix babylonica de Entre Ríos, Argentina. Se tomaron muestras a tres alturas en el fuste y en 9 posiciones radiales y se determinó la densidad básica y la morfometría de fibras y vasos. Los patrones de variación radial evidenciaron un leve aumento de magnitud con la edad, mientras que la mayor variación axial se manifestó mayoritariamente entre la base y la altura de $1,3 \mathrm{~m}$ el fuste, presentándose diferentes tendencias de acuerdo a la variable. Dependiendo de la variable analizada, la edad de transición entre madera juvenil y madura fue de 5 a 10 años o de 10 a 15 años. La longitud y ancho de fibras y el diámetro de vasos fueron los primeros parámetros en madurar. Los valores promedio de las variables de la madera juvenil y madura no mostraron diferencias importantes entre los leños, por lo tanto podrían considerarse como homogéneos desde el punto de vista anatómico y de densidad.

Palabras clave: Variación radial, regresiones segmentadas, morfometría de fibras, vasos, madera juvenil.

\section{ABSTRACT}

The aim of this work was to determine the patterns of axial and radial variation of the density and cellular morphometry of american willow wood, for determining the age of transition from juvenile to mature wood. The study was conducted on five trees of a 45-years-old plantation of a clone of Salix babylonica, from Entre Rios, Argentina. Samples were taken at three heights and at 9 radial positions of the stem for the determination of basic density and morphometry of fibers and vessels. The radial variation patterns showed a slight increase in magnitude with age, whereas the axial variation showed up between the base and a height of $1.3 \mathrm{~m}$ in the stem, presenting different tendencies according to the variable. Depending on the analyzed variable, the age of transition between juvenile and mature wood was 5 to 10 years, or 10 to 15 years. Fiber length and width and vessel diameter were the first characters to mature. The average values of the properties of juvenile and mature wood showed no substantial differences between logs, therefore they could be considered as anatomically and density homogeneous.

Key words: Radial variations, segmented regression, fibre properties, vessels, juvenile wood.

\footnotetext{
Facultad de Ciencias Agrarias y Forestales, Universidad Nacional de La Plata. La Plata, Argentina.

${ }^{2}$ Programa de Celulosa y Papel, Instituto de Materiales de Misiones, IMAM (UNaM-CONICET), Posadas, Argentina.

${ }^{3}$ Consejo Nacional de Investigaciones Científicas y Técnicas (CONICET), Buenos Aires, Argentina.

*Autor para correspondencia: cobasanaclara@yahoo.com.ar

Recibido: 05.03.2013 Aceptado: 10.11.2013
} 


\section{INTRODUCCIÓN}

La región más importante para la producción de salicáceas en Argentina, se ubica en el Bajo Delta del Paraná y alcanza una superficie cercana a 58.000 ha (Borodowski 2006). En el año 2010 se extrajeron en todo el país 3808571 toneladas de madera de distintas especies, de las cuales un $12 \%$ corresponde a sauces y álamos. En el caso particular del sauce, su principal uso es la producción de pulpa para papel periódico.

La calidad de madera destinada a proveer de material fibroso a la industria de la pulpa y el papel está determinada por la variabilidad de sus características físicas y químicas. Algunas de estas características son la densidad de la madera, la longitud de fibras, el espesor de pared de las mismas y la composición química (Barnett y Jeronimidis 2003). Las variables mencionadas de madera están influenciadas por la posición dentro del árbol (Zobel y Sprague 1998).

Se ha demostrado que existen patrones de variación radial (médula-corteza) y patrones de variación axial (base-ápice) en el fuste, asociados a diferentes edades fisiológicas del cambium y por lo tanto a la formación de madera juvenil y madura (Matyás y Peszlen 1997, Zobel y Sprague 1998, DeBell et al. 2002).

El leño juvenil, puede ser definido como la zona más cercana al centro del árbol, que se extiende desde la base hasta el ápice (Zobel y Talbert 1988). El tamaño del este cilindro central puede variar considerablemente de acuerdo a la propiedad estudiada y el método aplicado para determinar la edad de transición.

La tendencia general indica menores valores para ciertos atributos en los primeros anillos, incrementándose relativamente rápido durante algunos años, para luego estabilizarse o seguir aumentando gradualmente hacia el leño maduro (Bendtsen y Senft 1986).

Estas variaciones de las propiedades de la madera tienen un efecto importante sobre las características y el rendimiento de las pulpas y los productos del papel, así como también en la resistencia y la utilidad de sus productos sólidos (Downes et al. 1997).

Se han encontrado diferentes patrones de variación a lo largo del fuste en el género Salix para distintas propiedades, según los diferentes clones y los sitios de implantación en árboles con edades comprendidas entre 8-13 años cercanas al turno de corta de las plantaciones comerciales para papel en Argentina (Bonavía de Guth y Ragonese 1980, Monteoliva et al. 2002 a y b, 2005, Villegas y Marlats 2003, 2005, Monteoliva y Marlats 2007, Senisterra et al. 2005, Monteoliva 2011).

Particularmente para Salix babylonica var sacramenta "sauce americano", se reportaron patrones de variación que indican un aumento de la densidad básica de médula a corteza (Monteoliva y Marlats 2007) y un incremento de la densidad desde la base a la altura media del fuste y luego un descenso hacia el ápice (Monteoliva et al. 2002b, Villegas y Marlats, 2003, 2005, Monteoliva 2011). Para la misma especie, la longitud de fibra presenta un patrón de variación radial uniforme, aumentando con la edad (Bonavía y Ragonese 1980, Monteoliva et al. 2002a, Monteoliva 2011) y sin diferencias significativas a 3 alturas del fuste (Monteoliva et al. 2002a, Monteoliva 2011). Estas determinaciones se realizaron sobre ensayos experimentales de 13 años implantados en la misma localidad que los árboles del presente estudio (Entre Ríos, Argentina). No se encontraron trabajos que estudien las variaciones axiales y radiales del sauce americano en edades más avanzadas. Asimismo, las muestras fueron tomadas a una sola altura del fuste, generalmente a 1,3 m y considerando solamente dos o tres posiciones radiales, por lo tanto su diseño de muestreo no permitió analizar la transición de madera juvenil a madura. 
La edad de transición entre madera juvenil y madura es una característica de suma importancia que no ha sido determinada para el género Salix. El conocimiento de los patrones de variación axial y radial de las propiedades de las maderas y la zona de demarcación entre la madera juvenil y madura permitirá delimitar el turno de corta de acuerdo a la propiedad que se quiera potenciar y el destino final del producto a obtener. El conocimiento de la variación radial y axial de las distintas propiedades resultará también útil a la hora de definir la estrategia de muestreo y aportará datos que podrán ser utilizados en los proyectos de mejoramiento genético.

El objetivo de este trabajo fue analizar los patrones de variación axial y radial de la densidad básica y la morfometría de fibras y vasos para determinar la edad de transición de madera juvenil a madura en Salix babylonica.

\section{MATERIALES Y MÉTODOS}

Se estudiaron árboles de una plantación de Salix babylonica var sacramenta "sauce americano" procedentes del Establecimiento "Las Animas", Villa Paranacito, Entre Ríos ( $33^{\circ} 45^{\prime}$ LS; 59 05’ W) perteneciente a la empresa Papel Prensa S.A.

Se seleccionaron cinco árboles de 45 años de edad, según los protocolos de muestreo hallados en la bibliografía para estudios de variación radial y axial (Ting-Feng et al. 2006, Alves-Ramos et al. 2011) y los trabajos presentados por Hazman (1994) y Raymond (2006) sobre determinación del número de muestras. Se apearon y cortaron rodelas de $5 \mathrm{~cm}$ de espesor a tres alturas en el fuste: $0,3 \mathrm{~m} ; 1,3 \mathrm{~m}$ y 2,6 m. Las alturas seleccionadas para el estudio de variación axial se debieron a la forma forestal del árbol (altura comercial, altura de la primera ramificación y rectitud del tronco). Los sauces americanos presentan defectos de forma y alcanzan alturas comerciales de $7 \mathrm{~m}$ (Villegas y Marlats 2005, Villegas et al. 2009); asimismo, se encontró pudrición en las alturas superiores de los ejemplares una vez apeados, lo cual no permitió muestrear alturas superiores a los $3 \mathrm{~m}$.

De cada rodela se sacó una tabla central de $5 \mathrm{~cm}$ de ancho, en la cual se marcaron los anillos de crecimiento, previa preparación de la superficie transversal, con sucesivas lijas de diferentes granulometrías, hasta la perfecta visualización de los elementos celulares. Para la delimitación de los anillos, se utilizó una lupa binocular (10x). Sobre la tabla central, se marcaron las posiciones de muestreo radial, que se conformó uniendo 5 anillos de crecimiento. Según este criterio, se tomaron las probetas en 9 posiciones radiales por altura para la determinación de la densidad básica y para la elaboración de los macerados y preparados histológicos.

La determinación de densidad básica $\left(\mathrm{kg} / \mathrm{m}^{3}\right)$ se realizó según la norma IRAM 9544. Se registró el volumen saturado por inmersión total de la pieza en agua, y el peso anhidro en estufa a $102{ }^{\circ} \mathrm{C}+/-3$ ${ }^{\circ} \mathrm{C}$, pesado inmediatamente después de que la pieza alcanzara el equilibrio térmico con el ambiente. La densidad se determinó por el cociente entre el peso anhidro de la muestra y el volumen saturado de la misma.

Para la determinación de la longitud de fibras $(\mu \mathrm{m})$ se midieron 60 fibras $(\mathrm{n}=60)$ por rango de edad cambial sobre las 9 probetas radiales definidas anteriormente, extraídas a cada altura de muestreo del fuste. Las mediciones se realizaron sobre imágenes digitales tomadas con un microscopio óptico Olympus CX 31, previa maceración de las muestras según la técnica de maceración de Franklin (1945).

Las mediciones de lumen de fibras $(\mu \mathrm{m}, \mathrm{n}=300)$, ancho de fibras $(\mu \mathrm{m}, \mathrm{n}=300)$, espesor de pared $(\mu \mathrm{m}$, $\mathrm{n}=300)$, diámetro de vasos $(\mu \mathrm{m}, \mathrm{n}=500)$ y frecuencia de vasos $\left(\mathrm{n}^{\mathrm{o}} \mathrm{mm}^{-2}, \mathrm{n}=50\right.$ campos$)$ se realizaron sobre imágenes digitales microscópicas de cortes histológicos transversales teñidos con safranina en 5 posiciones radiales de muestreo y en todas las alturas. 
Los resultados se evaluaron estadísticamente mediante Análisis de la Varianza (ANOVA), considerando a la madera juvenil y madura, edad cambial y altura de muestreo, como fuentes de variación. El contraste de medias se realizó con la prueba de Tukey $(\mathrm{p}<0,05)$.

Para determinar la edad de transición de madera juvenil a madura, se utilizó el método de regresión segmentada sobre el patrón radial de la propiedad a analizar. Este método, supone que en el patrón radial de una propiedad dada (asociado con la edad del anillo de crecimiento) existe un cambio notorio en la pendiente de la línea de regresión de la misma, correspondiente a la edad de transición. El modelo de regresión por segmentos general, se presenta en la ecuación 1 y los modelos para las porciones de madera juvenil y madura en las ecuaciones 2 y 3 respectivamente (Tasissa y Burkhart 1998).

$$
\mathrm{Yi}=\beta_{0}+\beta_{1} \mathrm{x}_{\mathrm{i}}+\beta_{2}\left(\mathrm{x}_{\mathrm{i}}-\mathrm{T}\right) \mathrm{x}_{2}+\mathrm{e}_{\mathrm{i}}
$$

Donde:

$\mathrm{Yi}=$ característica de interés en el i-ésimo anillo de crecimiento

$\beta_{0}, \beta_{1}, \beta_{2}=$ coeficientes de regresión

$\mathrm{x}_{\mathrm{i}}=$ edad de formación del i-ésimo anillo de crecimiento

$\mathrm{T}=$ edad de transición de madera juvenil a madura

$\mathrm{x}_{2}=$ variable indicadora : $\mathrm{x}_{2}=0$ si $\mathrm{x}_{\mathrm{i}}<\mathrm{T}$ (madera juvenil) o $\mathrm{x}_{2}=1$ si $\mathrm{x}_{\mathrm{i}}>\mathrm{T}$ (madera madura)

$\mathrm{e}_{\mathrm{i}}=$ error aleatorio asociado al i-ésimo anillo de crecimiento.

De esta manera cuando $\mathrm{x}_{2}=0$ (madera juvenil):

$$
\mathrm{E}(\mathrm{Yi})=\beta_{0}+\beta_{1} \mathrm{x}_{\mathrm{i}}
$$

$\mathrm{y}$ cuando $\mathrm{x} 2=1$ (madera madura)

$$
\mathrm{E}(\mathrm{Yi})=\beta_{0}-\left(\beta_{2} \mathrm{~T}\right)+\left(\beta_{1}+\beta_{2}\right) \mathrm{x}_{\mathrm{i}}
$$

Se aplicó el método Piecewise Linear Regression (Breakpoint Regresión, Statistica v6). 


\section{RESULTADOS Y DISCUSIÓN}

\section{Variación radial y axial de las propiedades}

La edad cambial y la altura de muestreo en el fuste influyeron significativamente $(p<0,05)$ sobre todas las variables de la madera analizadas.

La tendencia de variación radial para la mayoría de las variables medidas consistió en un leve aumento de sus valores de médula a corteza, con diferentes fluctuaciones en edades intermedias. La frecuencia de vasos tuvo un comportamiento inverso, disminuyendo con la edad. En sentido axial, las principales diferencias se hallaron entre la base y la altura del pecho, presentándose diferentes tendencias de acuerdo a la variable (figura 1).
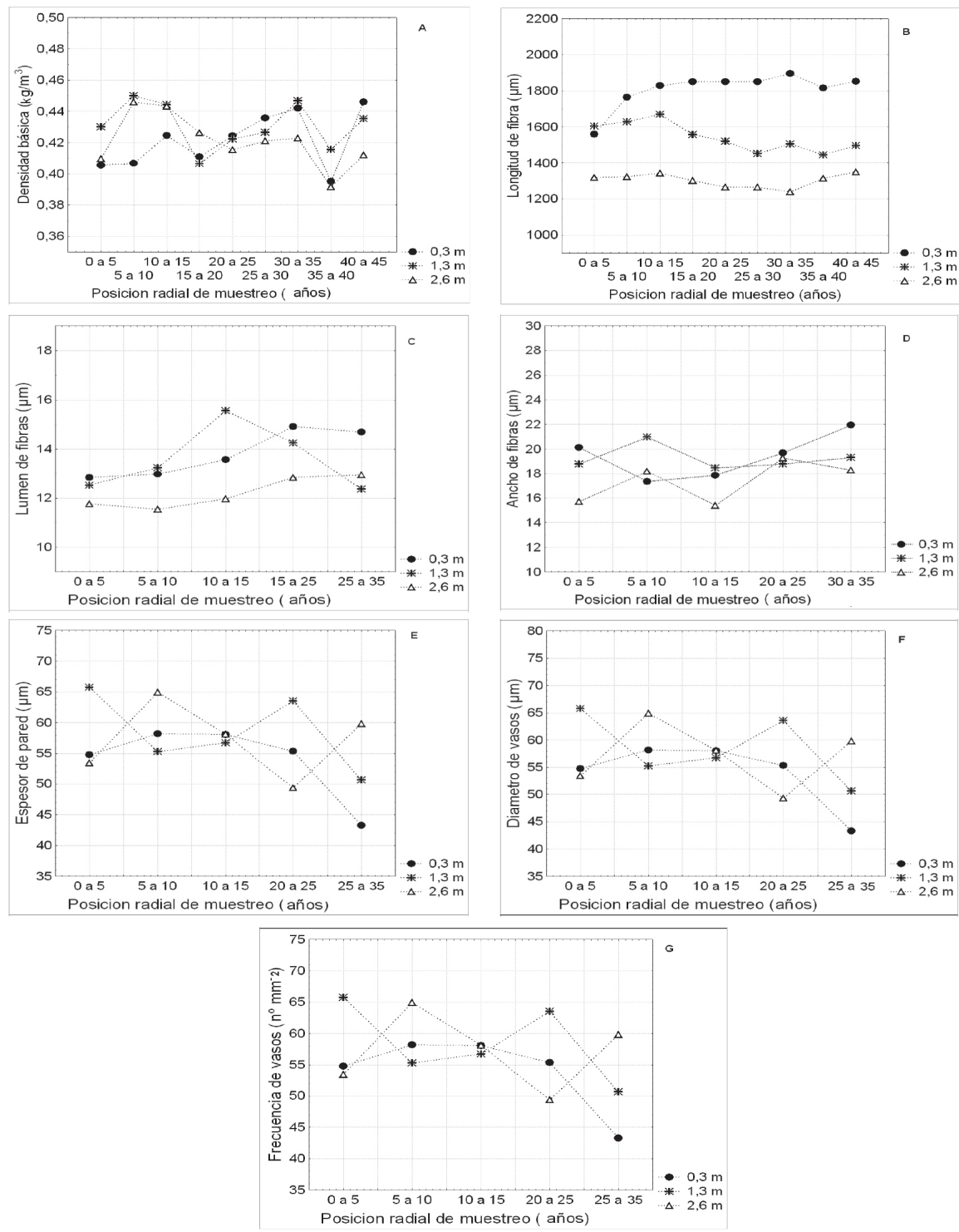

Figura 1. Patrones de variación axial y radial de las variables analizadas en la madera de sauce. 
Los valores de densidad básica se mantuvieron constantes, sin diferencias significativas entre si ( $>0,05)$ entre los rangos de edad 0 a 5 años y 30 a 35 años. A la edad de 35-40 años, los valores descendieron abruptamente en las 3 alturas de muestreo y luego aumentaron significativamente en el rango de edad de 40 a 45 años. Axialmente, la densidad básica no presentó variaciones significativas de sus valores promedio entre las 3 alturas (Figura 1A).

La longitud de fibra presentó diferentes patrones radiales según la altura de muestreo analizada. En la base $(0,3 \mathrm{~m})$, la longitud de fibras aumentó significativamente sus valores en los primeros rangos de edad ( 0 a 20 años) y luego se mantuvo constante. En la altura de 1,3m, presentó un aumento de los valores en las edades de 0-15 años y un posterior descenso hacia la corteza (sin diferencias significativas entre sí). En la altura superior $(2,6 \mathrm{~m})$, los valores fueron altos hasta los 20 años, descendieron significativamente hasta los 35 años y luego se estabilizaron con magnitudes semejantes a los primeros años. Axialmente, la longitud de fibra disminuyó significativamente con la altura (Figura 1B).

El lumen de fibra presentó un patrón de variación radial de aumento $(\mathrm{p}<0,05)$ de médula a corteza, siendo muy similar en las alturas de $0,3 \mathrm{~m}$ y $2,6 \mathrm{~m}$. A estas alturas, los valores promedios se separaron en dos grupos homogéneos de 0 a 15 años y de 20 a 35 años. La altura de 1,3 m, presentó un ascenso sostenido de los valores hasta los 15 años y luego un abrupto descenso. Al considerar la variación axial, el lumen disminuyó significativamente entre las primeras alturas y la altura de 2,6 m (Figura 1C).

Para el ancho de fibra, se halló una variación similar entre las alturas 1,3 m y 2,6 m, de aumento significativo hasta los 10 años, una disminución de los valores hasta los 15 años y luego una estabilización en magnitudes más altas hacia la corteza. En la base, se observó un descenso de las magnitudes hasta los 15 años, seguido de un aumento sostenido hacia las edades mayores. Dependiendo de la posición radial, la tendencia axial del ancho de fibras fue de un descenso de los valores con la altura de muestreo o no presentó diferencias significativas. En general la altura de 2,6 m fue la que presentó los menores valores (Figura 1D).

La tendencia radial para el espesor de pared, fue diferente según la altura en el fuste. Para la base los valores descendieron hasta los 10 años, aumentaron significativamente a los 15 años y se mantuvieron constante hacia la corteza. Para las otras dos alturas, las fibras aumentaron significativamente sus espesores de pared hasta los 10 años, luego disminuyeron sus valores hasta los 15 o 25 años (en la altura $2,6 \mathrm{~m}$ y $1,3 \mathrm{~m}$ respectivamente) y aumentaron nuevamente hacia la corteza (Figura 1E). La variación axial, presentó diferentes tendencias según la posición radial considerada, aunque podría considerarse a la base como la altura que presentó los menores valores.

El diámetro de vasos aumentó significativamente hasta la edad de 15 años, en todas las alturas de muestreo, luego disminuye con una estabilización de los valores hacia la corteza. Al considerar la variación axial, el diámetro de vasos a los 1,3 m presentó el menor valor, mostrando diferencias significativas en algunas posiciones con las alturas de $0,3 \mathrm{~m}$ y $2,6 \mathrm{~m}$ (Figura $1 \mathrm{~F}$ ).

La frecuencia de vasos no presentó diferencias significativas en las posiciones radiales a la altura de $0,3 \mathrm{~m}$. En los 1,3 $\mathrm{m}$ los vasos disminuyeron su frecuencia hasta los 15 años, aumentaron entre los 20-25 años y luego volvieron a descender hacia la corteza. En la parte superior del fuste, los valores aumentaron hasta los 10 años, disminuyeron en forma sostenida hasta los 25 años y luego aumentaron hacia la corteza. La frecuencia de vasos aumentó significativamente con la altura en el fuste en algunas posiciones radiales (Figura 1G).

Los patrones radiales generales de variación de las diferentes variables analizadas presentaron un leve aumento con la edad (excepto en el caso de la frecuencia de vasos) coincidentes con la bibliografía de sauces (Bonavía de Guth y Ragonese 1980) y de otras Salicáceas como diferentes especies de Populus (Matyás y Peszlen 1997, DeBell et al. 2002). 
En el género Salix a nivel nacional, se han publicado numerosos trabajos que involucran la calidad de madera para la producción de papel de diario, en los cuales se desarrollan diferentes patrones de variación a lo largo del fuste (Bonavía de Guth y Ragonese 1980, Monteoliva et al. 2002 a y b, 2005, Monteoliva y Marlats 2007, Senisterra et al. 2005, Villegas y Marlats 2003, 2005, Monteoliva 2011). Según estos estudios, los patrones de variación no son iguales en todos los clones y sitios. Sin embargo, estos estudios se realizaron sobre árboles con edades inferiores a las analizadas en este trabajo, por lo tanto las tendencias en la madera madura no han sido registradas.

Particularmente, para la especie en estudio, sauce americano, Monteoliva y Marlats (2007) estudiando árboles de 12 años provenientes del mismo sitio que los sauces de este trabajo y sobre la altura del pecho $(1,3 \mathrm{~m})$, publicaron un aumento de la densidad básica y longitud de fibras de médula a corteza, coincidente con lo hallado en las primeras posiciones radiales (15 años) del presente trabajo.

La variación de las dimensiones celulares de médula a corteza, es evidente en los parámetros que cambian sus dimensiones luego de la división cambial y se alargan axialmente como la longitud de traqueidas en coníferas y la longitud de las fibras y vasos en latifoliadas. Existe menos evidencia e investigación sobre la variación de las dimensiones celulares en el sentido transversal (ancho de fibras, lumen y espesor de pared, diámetro de vasos) (Panshin y de Zeeuw 1980). Se han publicado trabajos que demuestran que la longitud final de las fibras de latifoliadas está determinada en un $50 \%$ por la longitud de las iniciales cambiales y el otro $50 \%$ se debe a la elongación posterior que sufren durante la maduración (Ridoutt y Sands 1993). En este sentido, las variaciones de las dimensiones celulares halladas en este trabajo ponen en evidencia la marcada influencia de la edad cambial y la elongación posterior, sobre todo en la longitud de fibras.

La densidad es una propiedad compleja de la madera, dado que es la resultante de una combinación de características estructurales, cada una de la cuales tiene un sólido patrón hereditario propio. Está determinada principalmente por el tamaño y grosor de las paredes de las células de las fibras leñosas y también influyen la presencia de vasos y radios así como el volumen relativo de los diferentes tipos celulares (Zobel y Talbert 1988, Panshin y de Zeeuw 1980). Se asume que la densidad de la pared celular es constante en las especies y se calculó un valor promedio de $1500 \mathrm{~kg} / \mathrm{m}^{3}$ para latifoliadas (Barnett y Jeronimidis 2003). Por lo tanto, las variaciones en la densidad de la madera con las especies, se deben a la cantidad de pared celular presente en una unidad de volumen. En especies de porosidad difusa como los sauces, se presentan vasos de diámetro similares en todo el anillo de crecimiento y la cantidad de pared celular presente (y por lo tanto la densidad), estará afectada por los otros componentes anatómicos mayoritarios que son las fibras y el espesor de su pared celular (Barnett y Jeronimidis 2003). Teniendo en cuenta la variación menos marcada de las dimensiones celulares transversales medidas en esta especie (fibras y vasos), se observa que el comportamiento de la densidad refleja esa tendencia, siendo su variación radial de leve ascenso con la edad.

Los patrones axiales no presentaron un patrón uniforme en todas las variables analizadas. Este resultado coincide con la bibliografía de variación axial hallada para Salicáceas. Díaz et al. (2010) en su trabajo sobre la especie Populus deltoides "Australia 129-60" de 10 años de edad (de plantaciones comerciales en el Delta del Río Paraná, Argentina) publicaron que la densidad aumentó desde la base al ápice con un descenso de los valores en la altura de pecho. En el género Salix, Bonavía de Guth y Ragonese (1980) y Monteoliva et al. (2002b), no encontraron diferencias significativas de densidad a medida que aumenta la altura de muestreo en siete clones analizados de 13 años de edad, implantados en el mismo sitio de este trabajo (Villa Paranacito, Entre Ríos), coincidiendo con los resultados hallados en el presente estudio. Sin embargo, para Sauce americano, Salix nigra y Salix cv 13-14, estos mismos autores reportaron otras tendencias pero en porciones altas del fuste no alcanzadas por el diseño de muestreo del presente trabajo. 
La escasa información sobre la variación axial de la longitud de fibras conlleva a no poder definir un patrón de variación general. Estudios realizados sobre este tema (Novaresi et al. 1999, Monteoliva et al. 2005) reportan un patrón no uniforme de variación y generalmente sin diferencias significativas entre las alturas muestreadas. Sin embargo, para diferentes clones de Salix de 13 años de edad implantados en Entre Ríos, Argentina, y considerando 3 alturas de fuste (1,3; 4,3 y 6,5 m) muy superiores a las del presente trabajo, Monteoliva y Marlats (2007) reportaron una disminución de la longitud de fibras de la base al ápice, coincidiendo con nuestros resultados. Para el sauce americano se han reportados diferentes tendencias de variación axial de la longitud de fibra. En algunos trabajos no se observaron diferencias significativas entre las alturas estudiadas (Monteoliva et al. 2002a), mientras que en otros se observó un aumento desde la base hacia la altura media y luego una disminución hacia el ápice (Novaresi et al. 1999), aunque las alturas referidas en estos trabajos fueron también muy superiores a los del presente estudio.

No se hallaron referencias de análisis axiales de las otras variables anatómicas para el genero Salix.

Puede indicarse que los cambios hallados en el sentido axial dentro del fuste (considerando las limitaciones que impuso el muestreo del presente trabajo), son de menor magnitud que los hallados en sentido radial, tal como lo mencionaron otros autores para las latifoliadas de porosidad difusa (Wilkes 1988, Downes et al. 1997).

\section{Edad de transición}

Utilizando el método de regresión segmentada, la edad de transición entre madera juvenil (MJ) y madura (MM) presentó diferentes valores entre los rangos de 5 a 10 y de 10 a 15 años, dependiendo de la variable analizada (vasos, fibras o densidad). Los resultados se presentan en la tabla 1.

Tabla 1. Edad de transición (años) entre madera juvenil y madura para las propiedades medidas en madera de Salix babylonica en 3 alturas del fuste.

\begin{tabular}{|c|c|c|c|c|c|c|c|}
\hline \multirow[b]{2}{*}{$\begin{array}{c}\text { Altura } \\
(\mathrm{m})\end{array}$} & \multirow[b]{2}{*}{ Densidad } & \multicolumn{4}{|c|}{ Fibras } & \multicolumn{2}{|c|}{ Vasos } \\
\hline & & Longitud & Lumen & Espesor & Ancho & Diámetro & Frecuencia \\
\hline 0,3 & 10 a 15 & 10 a 15 & 15 a 20 & 10 a 15 & 5 a 10 & 10 a 15 & 10 a 15 \\
\hline 1,3 & 10 a 15 & 5 a 10 & 10 a 15 & 10 a 15 & 10 a 15 & 5 a 10 & 10 a 15 \\
\hline 2,6 & 15 a 20 & 10 a 15 & 15 a 20 & 10 a 15 & 10 a 15 & 10 a 15 & 10 a 15 \\
\hline
\end{tabular}

La edad de demarcación entre madera juvenil y madura, no fue la misma en cada variable y en cada altura de muestreo. Esto indica que el control fisiológico del proceso de maduración de las variables anatómicas, está determinado por el desarrollo del tronco y de la copa durante el crecimiento del árbol (Zobel y Sprague 1998). Los resultados indican que los primeros parámetros que alcanzaron la maduración, fueron la longitud, ancho de fibras y el diámetro de vasos (5-10 años), y dicha información puede ser importante para definir edades de rotación de una plantación para los diferentes destinos industriales (triturado o aserrado).

Aplicando el mismo modelo estadístico, Matyás y Peszlen (1997) hallaron edades de transición muy semejantes a las de este trabajo, estudiando 3 clones de Populus $x$ euroamericana de 10 y 14 años, a una única altura en el fuste, en dos sitios de Hungría. De la misma forma, hallaron que para diferentes variables anatómicas las edades de transición variaron entre 4-6 años para ancho de fibras, área de pared y diámetro de vasos, y entre 9-10 años para longitud de fibras. Demostraron también que el sitio influyó en la edad de maduración (pero no en el orden de maduración), ya que los valores medios se modificaron al determinarlos en árboles implantados en otro sitio. 
Teniendo en cuenta la densidad como propiedad, Bendtsen y Senft (1986) determinaron que el punto de demarcación entre madera juvenil y madura en Populus deltoides de 30 años en USA, se encontraba alrededor de los 17 a 18 años. Para longitud de fibras y ángulo microfibrillar, los valores se estabilizaron dos años más tarde.

No se hallaron antecedentes de determinación de edad de transición entre madera juvenil y madura en Salix.

Los valores promedio por tipo de madera juvenil o madura calculados para todas las alturas se presentan en el tabla 2. Se tomó como edad límite entre leños a la edad de transición de 10-15 años, obtenida con mayor frecuencia por las regresiones (Tabla 2).

Tabla 2. Propiedades de la madera juvenil (MJ) y madura (MM) para Salix babylonica.

\begin{tabular}{lcccc}
\hline Propiedad & $\begin{array}{c}\text { MJ } \\
(\text { media })\end{array}$ & $\begin{array}{c}\text { MJ } \\
(\mathrm{DS})\end{array}$ & $\begin{array}{c}\text { MM } \\
(\text { media })\end{array}$ & $\begin{array}{c}\text { MM } \\
(\mathrm{DS})\end{array}$ \\
\hline Densidad $\left(\mathrm{kg} / \mathrm{m}^{3}\right)$ & $414 \mathrm{a}^{*}$ & 10 & $424 \mathrm{a}$ & 10 \\
Longitud de fibra $(\mu \mathrm{m})$ & $1623 \mathrm{a}$ & 32,51 & $1618 \mathrm{a}$ & 12,22 \\
Ancho de fibra $(\mu \mathrm{m})$ & $17,8 \mathrm{a}$ & 1,68 & $19,1 \mathrm{a}$ & 0,56 \\
Lumen $(\mu \mathrm{m})$ & $12,8 \mathrm{a}$ & 1,08 & $13,8 \mathrm{a}$ & 1,12 \\
Espesor de pared $(\mu \mathrm{m})$ & $3,25 \mathrm{a}$ & 0,20 & $4,48 \mathrm{a}$ & 0,37 \\
Diámetro de vasos $(\mu \mathrm{m})$ & $65,6 \mathrm{a}$ & 2,05 & $64,1 \mathrm{a}$ & 5,05 \\
Frecuencia de vasos $\left(\mathrm{n}^{0} \mathrm{~mm}^{-1}\right)$ & $59,9 \mathrm{~b}$ & 1,61 & $53,5 \mathrm{a}$ & 5,11 \\
\hline
\end{tabular}

*Las letras indican las diferencias entre medias para MJ y MM según el test de Tukey $(\mathrm{p}<0,05)$.

En sauce americano, la madera juvenil presentó mayor frecuencia de vasos que la madera madura. Las demás variables anatómicas y densidad, no mostraron diferencias significativas. Estos resultados indican que la madera juvenil no es inferior en calidad a lo largo de todo su cilindro central, como se considera generalmente en la bibliografía (Bendtsen y Senft 1986, Matyás y Pezslen 1997, Zobel y Sprague 1998).

El volumen por árbol correspondiente a cada tipo de madera, sería de 22\% MJ y $78 \%$ MM en árboles de 45 años. En el caso de turnos de rotación cortos (10-12 años, utilizados en la industria papelera), y según las tendencias que marcan este estudio, el $82 \%$ del volumen sería madera juvenil.

\section{CONCLUSIONES}

El Salix babylonica (sauce americano) presenta una tendencia de variación radial en la mayoría de sus propiedades, que consiste en el leve aumento de sus valores de médula a corteza reproduciendo las tendencias radiales reportadas por diversos autores para las latifoliadas de porosidad difusa.

Según el esquema de muestreo planteado en este estudio, las principales diferencias en sentido axial, se producen entre la base y la altura del pecho dentro de la primera troza comercial.

Dependiendo de la variable analizada, la edad de transición entre madera juvenil y madura presentó diferentes valores entre los rangos de 5 a 10 y 10 a 15 años. La longitud y ancho de fibras y el diámetro de vasos fueron los primeros parámetros en madurar (5 a 10 años) mientras que el resto tuvo como edad de transición el rango de 10 a 15 años. 
Los valores promedio de las propiedades de la madera juvenil y madura indican que los dos tipos de leño no presentan diferencias importantes, por lo que podrían considerarse como homogéneos desde el punto de vista anatómico y de densidad.

Los resultados obtenidos, marcan una tendencia que debe ser analizada profundamente para otros clones de sauces y sitios de implantación. En este sentido, este estudio presenta una línea de exploración con posibilidades de aplicación, para el buen aprovechamiento de la madera según calidades y los requisitos de la industria consumidora.

\section{AGRADECIMIENTOS}

A Papel Prensa S.A. (Argentina), por facilitar el acceso al establecimiento y financiar el muestreo.

\section{BIBLIOGRAFÍA}

Alves-Ramos, L.; Figueiredo-Latorraca, J.: Scaramussa-Pastro, M.; Teixeira de Souza, M.; Garcia, R.; Monteiro de Carvalho, A. 2011. Variação radial dos caracteres anatômicos da madeira de Eucalyptus grandis W. Hill Ex Maiden e idade de transição entre lenho juvenil e adulto. Sci For Piracicaba 39 (92): 411-418.

Barnet, J.R.; Jeronimidis G. 2003. Wood quality and its biological basis. CRC Press. 226pp.

Bendtsen, B.; Senf, J. 1986. Mechanical and anatomical properties in individual growth rings of plantation-grown eastern cottonwood and loblolly pine. Wood and Fiber Science 18 (1): 23-38.

Bonavía de Guth, E.; Ragonese, A. 1980. Evaluación de las características del leño en relación a la calidad del papel de algunos híbridos de sauces obtenidos en Castelar (INTA). IDIA 393-394: 25-30.

Borodowski, E. 2006. Álamos y sauces en el Delta del Paraná: situación del sector y silvicultura. Actas de las I Jornadas Argentinas de Salicáceas, Buenos Aires, Argentina. pp. 61-70.

DeBell, D.S.; Singleton, R.; Harrington, C.A.; Gartner, B.L. 2002. Wood density and fiber length in young Populus stem: relation to clone, age, growth rate, and pruning. Wood and Fiber Science 34: 529-539.

Diaz, G., Monteoliva, S.; Alvarez, J.; Fernández Tschieder, E. 2010. Populus deltoides 'Australiano 129/60': variación axial de la densidad y desarrollo de un modelo predictivo de la densidad del árbol completo. Bosque 31 (1): 65-72.

Downes, G.; Hudson, I.; Raymond, C.; Dean, A.; Micheli, A.; Schimlek, L.; Evans, R.; Muneri, A. 1997. Sampling Eucalyptus for wood and fiber properties. Australia. CSIRO Publishing. 132p.

Franklin, G.L. 1945. Preparation of thin sections of synthetic resins and wood-resin composites, and a new macerating method for wood. Nature 155: 51.

Hazman, K.; Lewark, S. 1994. Sampling for wood properties in trial plots of 4 Eucalyptus species at Ruvu, Tanzania. Ann Sci For 51: 233-240. 
IRAM 9544. Instituto Argentino de Normalización. 1973. Norma IRAM 9544: Maderas. Método de determinación de la densidad aparente. Buenos Aires, Argentina.

Matyás, C.; Peszlen, I. 1997. Effect of age on selected wood quality traits of poplar clones. Silvae Genetica 46 (2-3): 64-72.

Monteoliva, S.; Senisterra, G.; Marquina, J.; Marlats, R. 2002a. Clones de sauce: longitud de la fibra en su madera. Rev FCA UNCuyo XXXIV(2): 49-56.

Monteoliva, S.; Senisterra, G.; Marquina, J.; Marlats, R.; Villegas, S. 2002b. Estudio de la variación de la densidad básica de la madera de ocho clones de sauce (Salix spp.). Revista FCAyF La Plata 105 (1): 29-34.

Monteoliva, S.; Senisterra, G.; Marlats, R. 2005. Variation of wood density and fiber length in six willow clones (Salix spp). IAWA Journal 26 (2): 197-202.

Monteoliva, S.; Marlats, R. 2007. Efecto del sitio, clon y edad sobre el crecimiento y la calidad de madera en sauces de corta rotación. Revista Investigación Agraria: Sistemas y Recursos Forestales $16(1): 15-24$.

Monteoliva, S. 2011. Madera de sauces para la producción de papel periódico. Editorial Académica Española.

Novaresi, M.; Delorenzi, F.; De Rosa, G.; Cervantes, P.; Rozas, C. 1999. Aptitud de Salicáceas para la industria del papel. CICELPA. Informe Técnico n OT 18-2927, 19p.

Panshin, A.; de Zeeuw, C. 1980. Textbook of wood technology. McGraw-Hill Book Company. 722p.

Raymond, C. 2006. Density assessment of radiata pine: Sampling strategy revisited. Holzforschung 60: $580-582$.

Ridoutt, B.; Sands, R. 1993. Within-tree variation in cambial anatomy and xylem cell differentiation in Eucalyptus globulus. Trees 8: 18-22.

Senisterra, G.; Ducid, M.; Marlats, R. 2005. Variación de la densidad básica de la madera en clones del género Populus. 3er Congreso Forestal Argentino y Latinoamericano. Corrientes, Argentina.

Tasissa, G.; Burkhart, H. 1998. Juvenil-mature wood demarcation in loblolly pine trees. Wood and Fiber Science 30 (2):119-127.

Ting-Feng, Y.; Braun, J.; Goldfarb, B.; Chang, H.; Kadla, J. 2006. Morphological and chemical variations between juvenile wood, mature wood, and compression wood of loblolly pine (Pinus taeda L.). Holzforschung 60: 1-8.

Villegas, M.S.; Marlats, R. 2003. Densidad de la madera: variación axial y radial en dos clones de Salix sp. X Jornadas Técnicas Forestales Ambientales FCFINTA, El Dorado. Argentina.

Villegas M.S.; Marlats, R. 2005. Altura de extracción de la muestra para la evaluación de densidad básica y blancura en madera de Salix sp. Bosque 26 (3): 121-132. 
Villegas, M.S.; Area, M.C.; Marlats, R. 2009. Caracterización de la madera de Salix. 1: Influencia del sitio, clon, edad y altura de muestreo. Revista de Investigación Agraria: Sistemas y Recursos Forestales 18(2): 192-203.

Wilkes, J. 1988. Variations in wood anatomy within species of Eucalyptus. IAWA Journal 9 (1): 13-23.

Zobel, B.J.; Talbert, J. 1988. Técnicas de mejoramiento genético de árboles forestales. Editorial Limusa, México. 385p.

Zobel, B.J.; Sprague, J.R. 1998. Juvenile wood in forest trees. Springer-Verlag, Berlín. 308p. 